\title{
Does Unnecessary Elective Neck Treatment Affect the Prognosis of No Laryngeal Cancer Patients?
}

\author{
ANTONIO SARNO, CORSO BOCCIOLINI, ALBERTO DEGANELLO*, SALVATORE COSCARELLI \\ and ORESTE GALLO
}

From the Institute of Otolaryngology - Head and Neck Surgery, University of Florence, Florence, Italy

\begin{abstract}
Sarno A, Bocciolini C, Deganello A, Coscarelli S, Gallo O. Does unnecessary elective neck treatment affect the prognosis of NO laryngeal cancer patients? Acta Otolaryngol 2004; 124: 980-985.

Objective-Among detractors of elective neck (N0) treatments, most suggest that lymphadenectomy removes a barrier to the spread of disease with deleterious immunologic consequences. To test this hypothesis we performed a retrospective comparison of the survival results of $\mathrm{N} 0$ laryngeal cancer patients who received unnecessary elective neck treatments $(\mathrm{N} 0-$ ) and those of N0 patients subjected to close and regular follow-up.

Material and Methods-A retrospective chart review of 749 N0 laryngeal cancer patients treated at the Institute of Otolaryngology - Head and Neck Surgery, University of Florence between January 1980 and January 1993 was performed. Of these, $245(33 \%)$ received elective neck dissection (ED), while the remaining $504(67 \%) \mathrm{N} 0$ patients were subjected to close and regular follow-up (wait-and-see policy; WS).

Results - Of the 245 ED patients, $43(17.5 \%)$ showed an occult neck disease, while of the 504 WS subjects, $83(17 \%)$ developed neck metastases during follow-up. Moreover, 15 ED patients subsequently experienced a contralateral occult failure despite a unilateral negative neck specimen (N0 - ). Thus, ultimately 187 ED (164 of whom were treated unilaterally and 23 bilaterally) and 421 WS patients were used for survival analysis. No differences in terms of postoperative complications, local or distant failure or disease-free or overall actuarial survival were found between the two groups analyzed.

Conclusions - These data indicate that unilateral or bilateral removal of cervical lymphatics in the absence of histologically proven lymph node metastases does not negatively affect the prognosis of N0 laryngeal cancer patients who were overtreated to the neck. Key words: laryngeal cancer, N0, neck surgery, prognosis, squamous cell carcinoma.
\end{abstract}

\section{INTRODUCTION}

In patients with squamous cell carcinoma of the larynx, and particularly of the supraglottis, management of the regional lymphatics is a crucial component of the overall treatment plan. If metastases to the cervical lymph nodes are clinically evident at diagnosis, treatment of the neck is mandatory. The situation is more controversial when the laryngeal cancer patient does not manifest clinical signs of neck disease (N0 patient). In this situation the physician must decide whether to perform elective treatment on the cervical lymph nodes or simply to wait for metastases to develop and then treat the patient when and if they occur. This issue, however, has yet to be resolved $(1,2)$.

At most institutions, a frequency of occult metastases exceeding $15-20 \%$ is considered to sufficient to justify elective neck therapy (2-6). Common arguments supporting this policy include the inevitable progression of occult nodal disease into clinically evident metastases requiring delayed therapeutic treatment of high-stage neck disease (7), the occurrence of inoperable neck disease in some $\mathrm{N} 0$ cases despite close

\footnotetext{
* Present address: Department of Otolaryngology — Head and Neck Surgery, VU University Medical Center, Amsterdam, The Netherlands.
}

and regular follow-up and, finally, the increased risk of the development of distant metastases in patients with untreated occult neck metastasis $(2-8)$. On the other hand, elective surgical treatment of the clinically negative neck in each instance of primary larynx carcinoma, obviously excluding T1 glottic tumors, could produce unnecessary morbidity, with higher medical costs and a longer hospital stay in a majority of patients $(70-80 \%)$ who are not likely to have cervical node metastases $(9,10)$. Furthermore, the appropriate use of ultrasound-guided fine-needle aspiration cytology seems to be more effective in controlling the great majority of neck disease in head and neck squamous cell cancer patients (1113). Moreover, it has been postulated that elective neck dissection removes a barrier to the spread of disease and also has a deleterious effect on the immune system because it eliminates sites of immune activation $(2,14)$. In agreement with a prognostic role of regional lymph node activation, improved survival has been linked with an active immunologic response detected in regional cervical lymph nodes from head and neck cancer patients in several studies (15-20). This hypothesis further supports the theory of immunological surveillance with respect to cancer (2123). In this regard, elective neck dissection could at least in theory weaken the host's mechanism(s) of 
recognition and elimination of neoplastic emboli from the primary tumor site and be responsible for regional and distant metastases $(14,15)$.

The aim of this study was to evaluate retrospectively the prognostic impact of unnecessary elective lymphadenectomy in $\mathrm{N} 0$ - laryngeal cancer patients. This hypothesis prompted us to compare the disease-free interval, actuarial and crude survival of two patient populations, both characterized by clinically negative neck at the initial diagnosis, the first of which received elective neck treatment and the second of which was subjected to close and regular follow-up.

\section{MATERIAL AND METHODS}

Our study population was selected from a database containing 749 previously untreated patients [723 men (96.5\%), 26 women (3.5\%); age range $47-79$ years; median age 64.5 years] with laryngeal squamous cell carcinoma and a clinically negative neck (N0) who underwent surgical treatment at the Institute of Otolaryngology - Head and Neck Surgery, University of Florence between January 1980 and January 1993. Of these 749 patients, $245(33 \%)$ received elective neck dissection (ED) as part of their primary treatment, while the remaining $504(67 \%)$ were subjected to close and regular follow-up, according to a wait-and-see policy (WS) (Fig. 1). The site and T-stage of the tumors according to the 1997 International Union Against Cancer guidelines are shown in Table I.

It has been suggested that elective neck dissection has a potentially detrimental prognostic significance in patients who do not have histologically positive occult lymph node disease $(\mathrm{N} 0-)$, in comparison with $\mathrm{N} 0$ patients who are not electively treated to the neck (wait-and-see policy). Therefore, we attempted to analyze the survival rates in these two groups, obviously excluding from both arms those laryngeal cancer patients who were experiencing occult neck disease, either histologically confirmed after an elective lymphadenectomy in the ED group or which occurred during follow-up in the absence of a local failure in the WS group. Therefore, the patients recruited were divided into two groups: (i) those with cancer of the larynx who had undergone an elective neck dissection with histologically negative neck (N0 - ; 202 ED patients); and (ii) those who had not undergone elective neck treatments without the clinical appearance of neck disease for at least 5 years of their follow-up (421 WS patients). None of these patients were submitted to adjuvant radiotherapy of the neck as empirical radiotherapy performed on a clinically negative neck would not have allowed evaluation of the development of occult cervical metastases, and thus would have led to the exclusion of these patients from the study group.

The criteria for selecting N0 laryngeal cancer patients for elective neck surgery were mostly subjective; however, an elective treatment was reserved for patients with advanced lesions (T3-4), supraglottic lesions, well-lateralized lesions involving peripheric laryngeal structures (which are usually at higher risk of occult node metastases), low-grade lesions and a short, fat neck leading to a difficult clinical examination. Conversely, a wait-and-see policy was employed in elderly patients or when the general conditions were poor, implicating high-risk surgical procedures.

In the ED group, 179 patients were treated with a unilateral elective lymphadenectomy and 23 with bilateral elective neck surgery. A functional neck dissection was the elective neck procedure performed unilaterally and bilaterally in all ED patients. Functional neck dissection was performed according to the technique proposed by Suàrez (24), with preservation of the spinal accessory nerve, sternocleidomastoid muscle and internal jugular vein (all these structures

Table I. Tumor stage and site distribution for the ED and WS groups. The values shown represent numbers of patients, with percentages in parentheses

\begin{tabular}{lccc}
\hline & ED group & WS group & Total \\
\hline T-stage & & & \\
T1 & $17(6.94)$ & $169(33.53)$ & $186(24.8)$ \\
T2 & $70(28.56)$ & $145(28.80)$ & $215(28.7)$ \\
T3 & $119(48.58)$ & $178(35.32)$ & $297(39.7)$ \\
T4 & $39(15.92)$ & $12(2.35)$ & $749(100)$ \\
Total & $245(100)$ & $504(100)$ & \\
Site & & & $372(49.67)$ \\
$\quad$ Supraglottic & $120(48.98)$ & $252(50.00)$ & $345(46.06)$ \\
Glottic & $107(43.67)$ & $238(47.22)$ & $32(4.27)$ \\
Subglottic & $18(7.35)$ & $14(2.78)$ & $749(100)$ \\
Total & $245(100)$ & $504(100)$ & \\
\hline
\end{tabular}




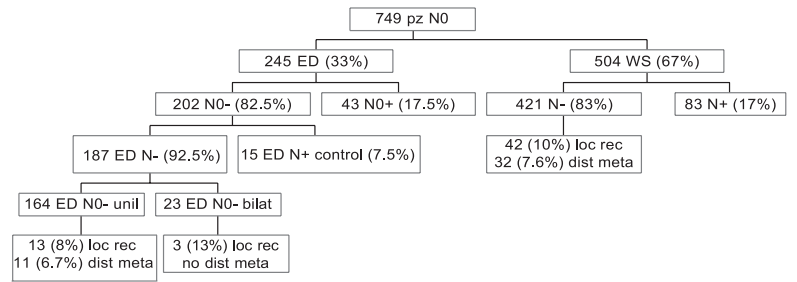

Fig. 1. Follow-up chart of the material, showing a comparison of local recurrences and distant metastases in the 187 ED N0 - and 421 WS N0 - patients.

are routinely removed by conventional radical neck procedures), but with removal of level II-V cervical lymph nodes.

Follow-up was at least 5 years or until death. A comparison was made between the two subsets of patients in terms of disease-free and overall survival. Considering the cause of death in this population, with particular reference to distant metastases and subsequent primary cancers, the criteria we used in particular for distinguishing lung metastases from primary laryngeal cancer and a second primary lung cancer were: (i) a period $>5$ years for the development of a lung lesion, in the presence of a squamous cell carcinoma; and (ii) different clinical and histopathological features.

To test the differences in our population we used Fisher's test. Considering the day of the initial surgery as the first day of observation, the disease-free and overall survival curves were calculated according to the Kaplan-Meier method (25).

$p<0.05$ was considered statistically significant. Statistical analysis was performed using the Stata (Stata Corporation, College Station, TX) and StatXact (Cytel, Cambridge, MA) programs.

\section{RESULTS}

Of the 749 N0 patients, 245 had an elective functional neck dissection and 504 were subjected to close and regular follow-up. Of the 245 ED patients, 43 $(17.5 \%)$ had clinically negative neck lymph nodes, although at the histopathological examination of neck specimens they showed histologically positive nodes $(\mathrm{N} 0+)$, while the remaining 202 patients $(82.5 \%)$ were histologically classified as having negative cervical lymph nodes $(\mathrm{N} 0-)$. Of the $43 \mathrm{~N} 0+$ patients, 21 $(48.8 \%)$ underwent a unilateral elective functional neck dissection, $13(30.3 \%)$ a bilateral functional neck dissection, $8(18.6 \%)$ a unilateral radical neck dissection and $1(2.3 \%)$ a radical neck dissection associated with a contralateral functional neck dissection.

Of the 504 WS patients, $421(83 \%)$ did not experience neck disease during follow-up, while the remaining $83(17 \%)$ had neck failure with the devel- opment of cervical metastases and were subsequently submitted to a therapeutic radical neck dissection. The median time to neck failure was 16 months (range 331 months).

Postoperative radiotherapy was performed to the neck in 5/43 (11.6\%) ED patients who proved to be $\mathrm{N} 0+$ after histopathological examination and in 17/83 $(20.5 \%)$ WS patients with neck failure. Doses ranged from 5000 to $6600 \mathrm{cGy}$ (median $5900 \mathrm{cGy}$ ).

Of the ED cases (202 patients) without occult neck disease, 15 developed lymph node metastases contralateral to the previously treated neck in the absence of local tumor recurrence. Most of these patients $(12 / 15 ; 80 \%)$ had supraglottic laryngeal carcinomas involving or extending up to the midline larynx. Therefore, it is conceivable that we detected occult contralateral neck metastases already present at the time of initial treatment (26). Although several nodal metastases or micrometastases may be missed by pathologists during routine histologic examinations (16), we assume that in such cases the initial elective unilateral neck treatment, which is unable to detect cancer cells in cervical lymphatics, did not have any prognostic impact on the development of contralateral occult disease. Therefore, these patients were further excluded from the ED group in which we tried to analyze only N0 laryngeal cancer patients without occult neck disease and for whom the neck surgery was unnecessary. Therefore, a total of 187 patients in the ED N0 group did not experience any neck failure during follow-up. Furthermore, this group was divided according to the type of elective neck procedure as follows: (i) 23 patients who underwent bilateral neck dissection; and (ii) 164 patients who underwent unilateral lymphadenectomy. According to these considerations, we tried to evaluate a possible prognostic impact of unnecessary lymphadenectomy performed in this group of 187 patients with clinically and histologically negative neck in comparison with the 421 N0 laryngeal cancer patients who were submitted to a wait-and-see policy and for whom any neck relapse was documented during follow-up.

Postoperative locoregional complications, including mainly wound infections, hemorrhages and skin fistulae, were documented in $35(18.7 \%)$ of the ED patients and $58(13.7 \%)$ of the N0 WS patients $(p=0.323)$.

Comparative analysis of local and distant relapse in the three groups (ED unilateral, ED bilateral, WS) did not show any statistically significant differences. In fact, we documented an almost analogous rate of local failure in the three groups $(8 \%, 13 \%$, and $10 \%$, respectively) ( $p=0.633)$ (Table II). Likewise, we did not find any statistically significant differences among the three groups analyzed in terms of distant metas- 
Table II. Distribution of local failures in 608 patients according to neck procedures. The values shown represent numbers of patients, with percentages in parentheses

\begin{tabular}{|c|c|c|c|c|}
\hline & N0 - ED unilateral & $\mathrm{N} 0-$ ED bilateral & N0 WS & Total \\
\hline \multicolumn{5}{|c|}{ Local failure } \\
\hline Yes & $13(8)$ & $3(13)$ & $42(10)$ & $58(10)$ \\
\hline No & $151(92)$ & $20(87)$ & $379(90)$ & $550(90)$ \\
\hline Total & 164 & 23 & 421 & 608 \\
\hline
\end{tabular}

Table III. Distribution of distant metastases in 608 patients according to neck procedures. The values shown represent numbers of patients, with percentages in parentheses

\begin{tabular}{|c|c|c|c|c|}
\hline & N0 - ED unilateral & N0 - ED bilateral & N0 WS & Total \\
\hline \multicolumn{5}{|c|}{ Distant metastases } \\
\hline Yes & $11(6.7)$ & $0(0)$ & $32(7.6)$ & $43(7)$ \\
\hline No & $153(93)$ & $23(100)$ & 389 (92.5) & $565(93)$ \\
\hline Total & 164 & 23 & 421 & 608 \\
\hline
\end{tabular}

tases $(6.7 \%, 0 \%$ and $7.6 \%$, respectively) $(p=0.375)$ (Table III). Moreover, there was no correlation between local recurrence and risk of distant disease, both overall and for each individual group $(p=0.629)$.

Actuarial analysis of survival did not show statistically significant differences between the selected groups in terms of disease-free or corrected survival $(p=0.490$ and 0.375 , respectively; log rank test) (Fig. 2). In addition, we performed a statistical analysis of survival according to tumor stage between ED and WS patients. Among patients with more advanced (T3-4) laryngeal cancers, a better prognosis, albeit statistically insignificant ( $p=0.11$; log rank test), was documented in the ED group.

\section{DISCUSSION}

The optimum management of the N0 neck in the patient with squamous cell carcinoma of the larynx

(A)

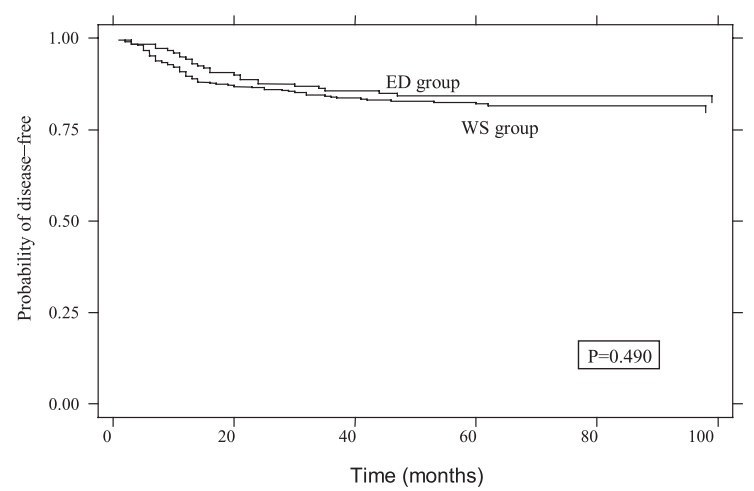

and upper aerodigestive tract remains controversial. Several authors (2-4) have shown a consistent rate of occult metastasis being present at diagnosis in these patients. In laryngeal cancer patients this rate varies according to the site and stage of the primary laryngeal tumor, being higher in supraglottic and advanced (T3-4) cancers (3-7), but $\approx 20-30 \%$ when all sites and stages are combined. A policy of elective neck treatment in these N0 patients will therefore result in overtreatment in almost $70-80 \%$ of cases, with unnecessary morbidity.

Advocates of observation of the N0 neck have also cited further reasons for this treatment philosophy, one of which involves avoiding the removal of a barrier to the spread of disease and the possible subsequent deleterious immunologic effect. In fact, within the complex interaction between tumor and host organism, draining of lymph nodes plays an important role in the organization and activation of an immune response induced by tumor antigens.

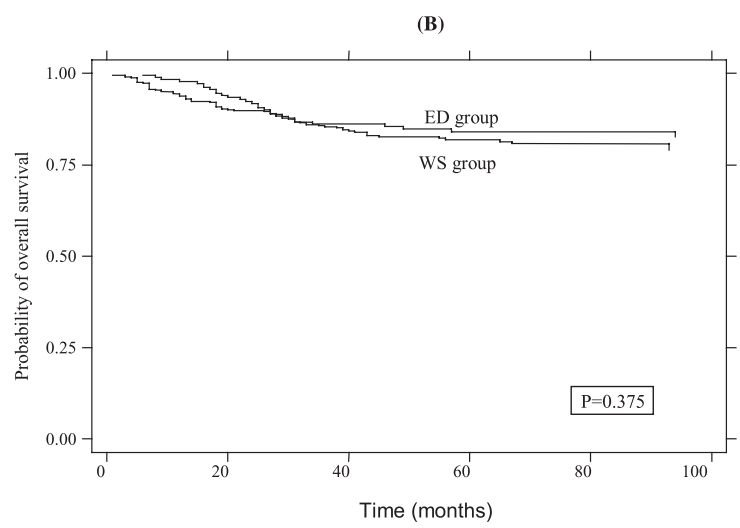

Fig. 2. (A) Disease-free and (B) overall survival curves for the ED and WS patients. 
In the past, several histomorphologic and, more recently, functional studies on immune cells from draining lymph nodes from laryngeal cancer patients with and without metastases have confirmed that an active immunologic response in regional lymph nodes takes place with possible prognostic significance (1418). Interestingly, some authors (27-29) demonstrated that, in metastatic lymph nodes, cancer cells seem to activate specific and non-specific immunosuppressive mechanism(s) when compared to unaffected cervical nodes from head and neck cancer patients. Moreover, we recently reported (19) that enlarged benign hyperplastic (false-positive) lymph nodes showing sinus histiocytosis were associated with better survival in patients with advanced laryngeal cancer when compared to those with analogous cancer but without clinical signs of lymph node enlargement electively treated to the neck. These studies suggest that the lymph nodes of the first defense row in the jugular chain are involved in an active immunological process in laryngeal cancer patients but that their colonization from neoplastic cells might be associated with impaired immune function $(14,27-29)$. Therefore, while it is possible to postulate that the surgical excision of draining lymph nodes in cancer patients might help the host immune system in the presence of nodal metastases, it would also seem that it would weaken activation of the host's defense mechanisms in the absence of tumor spread, by removing structures responsible for tumor antigen processing as well as immune cells. According to this hypothesis, this unnecessary and hypothetically deleterious elective neck treatment may be responsible for acute and late effects. The early effects may be correlated with an increase in locoregional postoperative complications, such as wound infection with delayed wound healing. However, it is difficult to demonstrate this because of the higher morbidity for patients undergoing neck dissection (due to increased operation time, related surgical stress and longer hospital stays) in comparison to those who were only treated for primary laryngeal cancer. Nonetheless, in our series we did not document any statistically significantly higher risk of postoperative complications in the ED group when compared with the WS patients who underwent laryngeal surgery only.

The late effects involve possible differences in terms of disease control and survival among patients electively overtreated in the neck and those who were simply observed because of host immunosuppression. Obviously, a well-planned prospective randomized trial could satisfactorily answer this question. However, the number of patients needed in order to yield statistically significant data is difficult to enroll, particularly by a single institution. In this study we retrospectively analyzed our own experience with 749 N0 laryngeal cancer patients, some of whom were overtreated electively (N0) and most of whom were being treated using a wait-and-see policy. We systematically excluded any patient who was submitted to radiation treatment on the neck, as it would not have been possible to detect the development of occult cervical node metastases in these patients.

Although prophylactic radiation treatment and elective prophylactic neck dissection are equivalent in controlling subclinical neck disease (30-33), we prefer the latter, as the current functional neck dissections and furthermore the selective neck dissections (34) have resulted in reduced local morbidity, lower medical cost and shorter hospitalization compared to complementary radiation treatment. In addition to this we are well aware of the local damage resulting from radiation therapy both locally (mucositis, xerostomia) and subsequently (endarteritis, radionecrosis, thyroid injury, radio-induced cancers). Furthermore, some authors (35) are still debating whether radiation therapy may have detrimental systemic effects, such as suppression of both the humoral and cellular arms of the immune system.

In conclusion, the results of this study offer evidence that unilateral or bilateral prophylactic neck dissection, when performed in patients without histologically proven neck disease, i.e. elective overtreatment, does not affect the prognosis of laryngeal cancer patients. In fact, besides neck control of the disease in patients given elective neck treatment or managed using a wait-and-see policy (an issue that was not addressed in this paper), we saw no differences in the risk of local and distant failure or in the outcome of the two analyzed groups. This contradicts one of the assumptions put forward by the proponents of neck observations and suggests that elective, possible selective (34) neck dissection is capable of accurately identifying laryngeal cancer patients with occult neck metastases at a low $\mathrm{N}$ stage with minimal morbidity and without detrimental prognostic effects in the overtreated patients (N0).

\section{REFERENCES}

1. Shah JP. Patterns of cervical lymph node metastasis from squamous carcinomas of the upper aerodigestive tract. Am J Surg 1990; 160: 405-9.

2. Snow GB. The N0 neck in head and neck cancer patients. Eur Arch Otorhinolaryngol 1993; 250: 423.

3. Bocca E, Calearo C, De Vincentiis I, Marullo T, Motta G, Ottavian A. Occult metastases of the larynx and their relationship to clinical and histological aspects of the primary tumor: a four-year multicentric research. Laryngoscope 1984; 94: 1086-90. 
4. Alajmo E, Aulisi L, Boccuzzi S. L'envahissement ganglionnaire dans les cancers du larynx: a propos des tumeurs N0. Cah ORL 1989; 10: 737-47.

5. Weber PC, Johnson JT, Meyers EN. Impact of bilateral neck dissection on pattern of recurrence and survival in supraglottic carcinoma. Arch Otolaryngol Head Neck Surg 1994; 120: 703-6.

6. Gallo O, Boddi V, Bottai GV, Parrella F, Fini Storchi O. Treatment of the clinically negative neck in laryngeal cancer patients. Head Neck 1996; 18: 566-72.

7. Andersen PE, Cambronero E, Shaha AR, Shah JP. The extent of neck disease after regional failure during observation of the neck. Am J Surg 1996; 172: 689-91.

8. Shah JP, Medina JE, Shaha AR, et al. Cervical lymph node metastasis. Curr Probl Surg 1993; 3: 273-344.

9. Skolnik EM, Katz AH, Becker SP. Evolution of the clinically negative neck. Ann Otol 1980; 89: 551-5.

10. Wing Yuen AP, Wei IW, Wong SFW. Critical appraisal of watchful waiting policy in the management of N0 neck of advanced laryngeal carcinoma. Arch Otolaryngol Head Neck Surg 1996; 122: 742-5.

11. Nieuwenhuis EJ, Castelijns JA, Pijpers R, et al. Waitand-see policy for the N0 neck in early-stage oral and oropharyngeal squamous cell carcinoma using ultrasonography-guided cytology: is there a role for identification of the sentinel node? Head Neck 2002; 24: 282-9.

12. Van den Brekel MW, Reitsma LC, Quak JJ, Smeele LE, Van der Linden JC, Snow GB. Sonographically guided aspiration cytology of neck nodes for selection of treatment and follow-up in patients with N0 head and neck cancer. AJNR Am J Neuroradiol 1999; 20: $1727-$ 31.

13. Van den Brekel MW, Castelijns JA, Reitsma LC, Leemans CR, Van der Waal I, Snow GB. Outcome of observing the N0 neck using ultrasonographic-guided cytology for follow-up. Arch Otolaryngol Head Neck Surg 1999; 125: 153-6.

14. Tachibana T, Yoshida K. Role of the regional lymph node in cancer metastasis. Cancer Metastasis Rev 1986; 5: $55-66$.

15. Ferlito A, Polidoro F. Biological and prognostic implications of the morphologic aspects of immune reaction in lymph nodes draining head and neck cancers. $\mathbf{J}$ Laryngol Otol 1979; 93: 153-75.

16. Schuller DE, Koolemans-Beynen AR, Libby DH, Rinehart $\mathrm{JJ}$, et al. Impact of metastases on nodal immunoreactivity in head and neck cancer. Laryngoscope 1986; 96: 1189-92.

17. Patt BS, Close LG, Vuitch F. Prognostic significance of sinus histiocytosis in metastatic laryngeal cancer. Laryngoscope 1993; 103: 498-502.

18. Klimek T, Glanz H, Dreyer T. Histomorphological characteristics of non-metastatic lymph nodes in patients with head and neck cancer according to the site in the neck. Acta Otolaryngol (Stockh) 1996; 116: 336-40.

19. Gallo O, Boddi V, Bottai GV, Franchi A, Fini Storchi O. Prognostic significance of clinically false positive cervical lymph nodes in patients with laryngeal carcinoma. Cancer 1995; 75: 1077-83.

20. Yuen AP, Wei WI, Wong SH. Critical appraisal of watchful waiting policy in the management of N0 neck of advanced laryngeal carcinoma. Arch Otolaryngol Head Neck Surg 1996; 122: 742-5.
21. Saleh FH, Crotty KA, Hersey P, Menzies SW, Rahman W. Autonomous histopathological regression of primary tumours associated with specific immune responses to cancer antigens. J Pathol 2003; 200: 383-95.

22. Hammad AM, Paris GR, van Heuven WA, et al. Spontaneous regression of choroidal metastasis from renal cell carcinoma. Am J Ophthalmol 2003; 135: 911-3.

23. Sanchez-Ortiz RF, Tannir N, Ahrar K, Wood CG. Spontaneous regression of pulmonary metastases from renal cell carcinoma after radio frequency ablation of primary tumor: an in situ tumor vaccine? J Urol 2003; 170: $178-9$.

24. Suàrez $\mathrm{O}$. El problema de las metàstasis linfàticas $\mathrm{y}$ alejadas del cancer de laringe i hipofaringe. Rev Otorrinolaringol (Santiago) 1963; 23: 83-99.

25. Kaplan EL, Meier P. Non parametric estimations for incomplete observations. J Am Stat Assoc 1956; 53: 457-81.

26. Gallo O, Fini-Storchi I, Napolitano L. Treatment of the contralateral negative neck in supraglottic cancer patients with unilateral node metastases (N1-3). Head Neck 2000; 22: 386-92.

27. Cozzolino F, Torcia M, Carossino AM, et al. Characterization of cells from invaded lymph nodes in patients with solid tumors. Lymphokine requirement for tumor-specific lymphoproliferative response. J Exp Med 1987; 166: 303-18.

28. Mickel RA, Kessler DJ, Taylor JM. Natural killer cell cytotoxicity in the peripheral blood, cervical lymph nodes, and tumor of head and neck cancer patients. Cancer Res 1988; 48: 5017-22.

29. Wang MB, Lichtenstein A, Mickel RA. Hierarchical immunosuppression of regional lymph nodes in patients with head and neck squamous cell carcinoma. Otolaryngol Head Neck Surg 1991; 105: 517-27.

30. Breau RL, Suen JY. Management of the N0 neck. Otolaryngol Clin North Am 1998; 31: 657-69.

31. Chow JM, Levin BC, Krivit JS, et al. Radiotherapy or surgery for subclinical cervical node metastases. Arch Otolaryngol Head Neck Surg 1990; 115: 981-4.

32. Fletcher GH. Subclinical disease. Cancer 1984; 53: 1274-84

33. Shasha D, Harrison LB. Elective irradiation of the N0 neck in squamous cell carcinoma of the upper aerodigestive tract. Otolaryngol Clin North Am 1998; 31: 803-13.

34. Spiro RH, Gallo O, Shah JP. Selective jugular node dissections in patients with pharyngeal and laryngeal squamous carcinoma. Am J Surg 1993; 166: 399-402.

35. Pillsbury HC, Clark M. A rationale for therapy of the N0 neck. Laryngoscope 1997; 107: 1294-315.

Submitted August 21, 2003; accepted December 18, 2003

Address for correspondence:

Oreste Gallo, MD

Istituto di Otorinolaringoiatria

Viale G.B. Morgagni n 85

IT-50134 Firenze

Italy

Tel.: + 39055411772

Fax: + 39055435649

E-mail: o.gallo@dfc.unifi.it 
Copyright of Acta Oto-Laryngologica is the property of Taylor \& Francis Ltd and its content may not be copied or emailed to multiple sites or posted to a listserv without the copyright holder's express written permission. However, users may print, download, or email articles for individual use. 